\title{
Sistemas de evaluación de la investigación: aproximación al modelo «performativo» británico o modelo basado en resultados
}

\author{
Ramón Martínez García \\ Personal investigador de la Facultad de Ciencias de la \\ Comunicación de la Universidad de Málaga y licenciado en Derecho

\section{Extracto}

La implantación de sistemas de evaluación y financiación de la investigación basados en resultados es una recomendación constante por parte de los organismos internacionales. Dentro del Espacio Europeo de Investigación (EEI), España es una excepción y no ha introducido medidas "performativas». El objetivo general de este trabajo es describir y analizar las características básicas de este sistema. Para ello se ha optado por realizar una aproximación al sistema británico, primer país en implantar estas políticas científicas, a partir de una investigación documental basada en la revisión de la literatura especializada, en combinación con la interpretación exegética de la norma. Los resultados hablan de un sistema basado en una lógica retributiva del sistema financiador. La evaluación de la investigación se basa en la revisión por pares con una introducción paulatina de las métricas como elemento complementario. Las conclusiones apuntan a la necesidad de seguir profundizando en el estudio de marcos comparativos que faciliten la transición entre un modelo institucional a otro basado en resultados.

Palabras clave: evaluación y financiación de la investigación; Reino Unido; research excellence framework (REF); revisión por pares informada; evaluación del impacto de la ciencia. 


\title{
Research evaluation systems: approach to the british performance-based research funding system
}

\author{
Ramón Martínez García
}

\begin{abstract}
The implementation of performance-based research funding system is a constant by international organizations. In the European Research Area (ERA), Spain is an exception and there have been no performative measures. The general objective of this work is to describe and analyze the basic characteristics of this system. For this purpose, we have chosen to approach the british system, the first country to implement these scientific policies, based on a documentary research based on the review of specialized literature in combination with the exegetical interpretation of the standard. The results speak of a system based on a retributive logic of the financing system. The evaluation of the research is based on peer review with a gradual introduction of the metrics as a complementary element. The conclusions point to the need to continue deepening the study of comparative frameworks that facilitate the transition between an institutional model to another based on results.
\end{abstract}

Keywords: perfomance-based research funding system; United Kingdom; research excellence framework (REF); informed peer review; impact case. research funding system. Tecnología, Ciencia y Educación, 14, 71-93. 


\section{Sumario}

1. Introducción

2. Metodología

3. Contexto

4. Aproximación al modelo de financiación de la investigación en el Reino Unido

5. Antecedentes del REF

6. REF 2014: la introducción de las métricas y la medición del impacto de la ciencia

7. Resultados y críticas

8. Conclusiones

Referencias bibliográficas

Nota: este trabajo se enmarca dentro del Proyecto de I+D+i del Programa Estatal de Generación de Conocimiento y Fortalecimiento Científico y Tecnológico (Subprograma Estatal de Generación de Conocimiento 2018), con referencia PGC2018-093358-B-100. 


\section{Introducción}

La implementación de políticas que optimicen el rendimiento de los sistemas de investigación es una prioridad para el EEI (Comisión Europea, 2017). La Organización para la Cooperación y el Desarrollo Económicos (OCDE) también ha remarcado la necesidad de incorporar sistemas de financiación de la investigación vinculados a los resultados (OCDE, 2010).

La importancia de crear estudios comparados en el marco de los sistemas de evaluación y financiación de la investigación estriba en que facilitan la homogeneización de estas políticas científicas en el ámbito del EEI.

En este estudio se realiza una aproximación al modelo británico, primer país del mundo en implantar sistemas de evaluación basados en resultados. Se ha construido desde un enfoque cualitativo de análisis basado en el trabajo de fuentes bibliográficas de diversa naturaleza: literatura académica clásica, documentos e informes sobre prácticas políticas y fuentes normativas.

Como se desarrollará a lo largo del estudio, la literatura anglosajona que trata el análisis de los sistemas «performativos» es amplia. Sin embargo, no existe ningún estudio en castellano que ponga el foco en el sistema de evaluación y financiación «performativo» británico para realizar un análisis en profundidad. El principal objetivo es indagar en los antecedentes históricos del sistema de evaluación de la investigación del Reino Unido para poder ofrecer un marco de comprensión y profundización sobre la actualidad de este modelo.

El modelo «performativo» de la evaluación de la investigación se caracteriza por vincular los rendimientos derivados de la investigación a incentivos económicos a través del modelo de financiación.

La principal característica es que la evaluación de los principales outputs de la investigación se realiza ex post (Hicks, 2012), mientras que, en el otro extremo, nos encontramos el modelo institucional, caracterizado por una financiación en bloque, de naturaleza no competitiva, y realizada ex ante (Zacharewicz, Lepori, Reale y Jonkers, 2019).

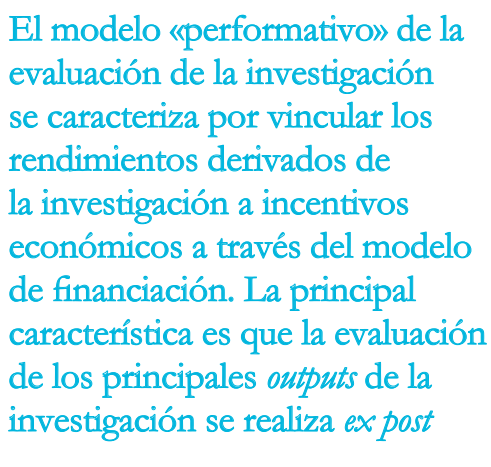

El modelo «performativo» de la evaluación de la investigación se caracteriza por vincular los rendimientos derivados de la investigación a incentivos económicos a través del modelo de financiación. La principal característica es que la evaluación de los principales outputs de la investigación se realiza expost 


\section{Metodología}

Esta investigación es de naturaleza documental. A nivel metodológico se ha basado en la combinación de una revisión bibliográfica de la literatura especializada con una investigación jurídica mixta (Witker, 2008; García, 2015). Desde el punto de vista del derecho, esta perspectiva metodológica es un proceso inductivo e interpretativo. Se observa la norma particular para, tras un proceso de interpretación, extraer conclusiones sobre la práctica discursiva institucional.

El punto de partida epistemológico es el paradigma sociocrítico (Denzin, 1970), que pretende ofrecer herramientas de análisis a la comunidad académica que permitan no solo reinterpretar el fenómeno, sino también participar activamente en la construcción de otros modelos científicos. Este rol activo de la investigación cualitativa se conoce como "octavo momento» (Denzin y Lincoln, 2011).

La justificación de este estudio viene derivada de la situación del Reino Unido como exponente, claro y consolidado, en la implementación de este tipo de políticas, siendo un país pionero en adoptar sistemas «performativos» de evaluación (Stern, 2016). Este hecho ha producido una literatura muy especializada. El análisis de esta literatura, en combinación con la interpretación de la norma, puede arrojar claves muy interesantes desde el punto de vista de los marcos comparativos de los sistemas de evaluación de la investigación (SEI).

\section{Contexto}

Los SEl pueden ser definidos como «instrumentos usados para certificar la calidad del conocimiento científico, a través de una evaluación cualitativa del contenido sustantivo de las contribuciones, pero, al mismo tiempo, están asociados a estímulos económicos y académicos para los científicos» (Osuna, 2010, p. 225).

En un contexto donde la cultura de la rendición de cuentas por parte de los poderes públicos respecto a la investigación está en crecimiento constante, existe una demanda institucional de SEl cada vez más precisos y efectivos, orientados a controlar, reconocer y premiar aquellos resultados que transformen la sociedad y la economía según los parámetros preestablecidos por los órganos evaluadores. 
Geuna y Martin (2003) aclaran que la evaluación sobre la investigación en el ámbito de la educación superior se ha convertido en un factor estratégico para el Estado, al objeto de satisfacer las demandas de rendición de cuentas, ya que se entiende la educación superior como una inversión, y reclaman conocer los resultados de esa inversión.

En el caso del Reino Unido, los SEl tienen una larga trayectoria, al ser un país pionero en incorporar este tipo de políticas científicas. Actualmente, sigue siendo líder en este campo, conocido como performance-based research system (evaluación basada en resultados o evaluación «performativa»). Desde su implementación en 1986 hasta 2014, fecha en la que se ha desarrollado el último ejercicio, ha pasado por diversas denominaciones:

- 1986 y 1989. Research selectivity exercise (RSE).

- 1992, 1996, 2001 y 2008. Research assessment exercise (RAE).

- 2014. Research excellence framework (REF).

La evaluación se basa en dos elementos fundamentalmente: los outputs y el sistema peer review, organizado en macropaneles de expertos. Por output se entiende cualquier producto derivado de la actividad investigadora y que pueda ser susceptible de ser evaluado. Esta amplitud incluye no solo producción escrita en el ámbito académico, sino también producción artística, exposiciones, creaciones audiovisuales, desarrollo de nuevos materiales y, en definitiva, cualquier producción derivada del trabajo como investigador (REF, 2019). Estos son preseleccionados por el personal investigador y expuestos a los responsables de su institución de educación superior. Después, será la propia institución la que decida cuál elegir como muestra representativa de su labor investigadora (REF, 2011).

Con el tiempo, se incorporaron otros elementos evaluables, como la transferencia de resultados, basado en la evaluación de casos de impacto (impact cases) ${ }^{1}$ o el ecosistema académico (research environment).

Teniendo en cuenta lo apuntado hasta ahora, este proceso se podría definir como un ejercicio evaluativo, sistemático y periódico, basado en el peer review, focalizado en medir la calidad de la investigación en una institución concreta al objeto de redistribuir los fondos públicos destinados a la investigación (Barker, 2007; Marginson, 2015).

1 Más adelante, en el epígrafe dedicado al REF 2014 se analiza con más detenimiento este concepto. Sirva ahora la definición ofrecida por la guía editada por el propio REF (2011):

Impact includes, but is not limited to, an effect on, change or benefit to the activity, attitude, awareness, behaviour, capacity, opportunity, performance, policy, practice, process or understanding of an audience, beneficiary, community, constituency, organisation or individuals in any geographic location whether locally, regionally, nationally or internationally. Impact includes the reduction or prevention of harm, risk, cost or other negative effects (p. 48). 


\section{Aproximación al modelo de financiación de la investigación en el Reino Unido}

La OCDE (2010) ha identificado de forma clara la necesidad de que los SEl estén basados en resultados, por lo que apunta claramente a este tipo de sistemas evaluativos como los más aconsejables y eficientes para la redistribución de recursos económicos para la universidad, en paralelo al modelo de financiación institucional tradicional, creando así un modelo de financiación dual, característico del Reino Unido.

Se le denomina «modelo dual de financiación» porque los fondos provienen de dos vías fundamentalmente:

- Una se refiere a la que proviene de los resultados en el REF.

- La otra proviene de los research councils (RC), que son organismos de naturaleza pública. Están divididos por áreas temáticas y cada uno goza de autonomía total.

Para el REF, la evaluación de la calidad es realizada mediante un análisis ex post sobre una muestra de la producción científica presentada por la institución que corresponda, mientras que los fondos provenientes de los diferentes RC tienen un marcado carácter prospectivo y son otorgados en función de la propuesta de proyectos ex ante projects funding). Esta forma de financiación de la investigación podría asemejarse a la que realiza la Agencia Estatal de la Investigación a través de los diferentes planes nacionales. El sistema de financiación dual combina métodos de asignación basados en resultados y en proyectos. La financiación que proviene del consejo de investigación es un ejercicio esencialmente prospectivo, ya que se basa en las actividades de investigación propuestas. Reflejarán inevitablemente la reputación de los investigadores que avalan la propuesta y se basarán en trabajos anteriores. En contraste, el elemento principal de la financiación basada en el REF se centra en una evaluación periódica sobre los resultados de la investigación (Hughes, Kitson, Bullock y Milner, 2013). Este sistema permite elaborar un mapa de redistribución de los fondos dirigidos a la educación superior basado en los resultados obtenidos en los ejercicios evaluativos (Barker, 2007).

\section{Antecedentes del REF}

En total se han realizado siete ejercicios evaluativos y, como veremos en el análisis, han recibido distintas denominaciones. Como ya hemos comentado anteriormente, en sus inicios, esta prueba recibió el nombre de RSE (1986 y 1989). Más adelante, pasó a conocerse como RAE (1992, 1996, 2001 y 2008). Y, finalmente, recibió el nombre de REF (2014) 2.

2 El próximo REF se celebrará en 2021. 
En $1984^{3}$, el University Grants Committee (UGC) 4 anuncia su intención de adoptar un sistema más selectivo de financiación que asegurase el buen aprovechamiento de los recursos públicos (Bauer y Kogan, 1997).

Como se indicó anteriormente, el primer ejercicio masivo de evaluación de la investigación en el Reino Unido se produjo en 1986 y fue conocido como RSE. El propósito inicial era recabar una muestra intencional representativa de la investigación realizada por la universidad para, una vez procesada y estandarizada, establecer 37 áreas de conocimiento que fueran evaluadas por comités de expertos. Cada universidad debía presentar ante el comité las mejores cinco publicaciones de los últimos cinco años en cada una de las áreas. Además, el RSE de 1986 tenía en cuenta el número total de estudiantes matriculados y las prioridades establecidas en la investigación a través de los planes estratégicos de cada universidad (Stern, 2016). Esta dinámica se irá depurando a los largo de los años, pero la base será la misma en todos los ejercicios posteriores.

Desde un primer momento, el RSE despertó fuertes críticas por parte de la comunidad académica, que lo vio como un ejercicio absurdo, falto de consistencia y con una forma inapropiada e imprecisa de recolección de datos (Gilroy

Desde un primer momento, el RSE despertó fuertes críticas por parte de la comunidad académica y McManara, 2009). En respuesta, el UGC se apresuró a realizar un segundo ejercicio nacional de evaluación en 1989 buscando mayor consenso dentro de la comunidad académica. EI RSE 1989 se caracteriza por haber sido diseñado con más transparencia, por haber formalizado el panel peer review como método fundamental de evaluación y por haber ampliado hasta 152 las áreas de conocimiento analizadas, de las 37 que inicialmente fueron previstas en el anterior ejercicio.

Estos dos primeros ejercicios piloto (RSE 1986 y 1989) sirvieron para distribuir una parte relativamente pequeña de fondos (Stern, 2016) y para ir asentando la cultura de la rendición de cuentas por parte de la comunidad académica.

En 1992 se crearon el HEFCE y el HEFCW. Este órgano pasó a ser el responsable de la distribución de los fondos públicos destinados a la educación superior (véase nota 4). El nuevo ejercicio de evaluación fue rebautizado como RAE. Se incrementó notablemente

3 Este cambio de modelo se produce en consonancia con el contexto político que caracteriza al Reino Unido en esta época (el Gobierno conservador, liberal y reformista de Margaret Thatcher [1979-1990]).

4 Este organismo asesoró al Gobierno británico en materia de financiación de la educación superior desde 1919 hasta 1989, fecha en la que se crea el Universities Funding Council (1989-1992). En 1992 se funda el Higher Education Funding Council for England (HEFCE), el Higher Education Funding Council for Wales (HEFCW) y el Scottish Funding Council (SFC). Estos organismos son los encargados de distribuir el dinero público en la educación superior. 
el porcentaje de financiación de la investigación en función de los resultados: prácticamente el $90 \%$ de la financiación de la investigación pasó a depender de los resultados del RAE (Stern, 2016).

Tras el RAE 1992, la comunidad académica ya había pasado tres procesos evaluativos integrales e iba asumiendo el nuevo paradigma de la rendición de cuentas respecto al Estado, principal actor financiador de la investigación. Algunos autores han criticado duramente el devenir de una nueva lógica comercial en el marco de la producción del conocimiento, producida a partir de la consolidación del sistema «performativo» de evaluación (Geuna y Martin, 2003).

En 1996 se realizó un nuevo RAE que, como los anteriores, tenía como principal objetivo elaborar clasificaciones de calidad para distribuir los fondos en función de los resultados. Este ejercicio fue precedido por extensas consultas a la comunidad universitaria sobre los puntos que había que mejorar en relación a los ejercicios anteriores. Una de las principales conclusiones a las que se llegó fue abandonar el paradigma de la cantidad como medida de la calidad, es decir, hasta el RAE 1996, la cantidad de outputs evaluables fue utilizada por los paneles peer review como una medida fundamental para medir la producción científica. A partir de este ejercicio, se dejó atrás esta concepción para centrarse en la calidad, analizada independientemente por el panel en cuestión, dejando de reclamar una lista completa de todos los outputs (Bence y Oppenheim, 2005). En este sentido, la propia guía elaborada por el HEFCE puntualizaba lo siguiente:

El HEFCE desea señalar claramente que el RAE está preocupado por la calidad de la investigación y que el número de publicaciones, y otras formas de resultados evaluables, no se consideran necesariamente un indicador de la calidad de la investigación (RAE, 1996b).

Otro de los cambios importantes fue la introducción del peer esteem, que podría ser traducido como un «indicador de reputación entre pares». Para ello, se valoraron las conferencias, los cursos, pertenecer a consejos editoriales o a colegios profesionales y los premios. Este indicador podría ser considerado como un antecedente de los actuales altmetrics, que pretenden medir el impacto entre pares a través de la combinación de indicadores relacionados con la difusión digital y la reputación.

Según el informe conocido como The Dearing Report, elaborado para la Comisión Nacional de Investigación del Reino Unido en 1997, el sistema de evaluación y financiación basado en resultados había quedado plenamente consolidado e integrado en las prácticas académicas. Hay que recordar que ya se habían celebrado cuatro evaluaciones generales (1986, 1989, 1992 y 1996).

Desde la perspectiva de la política institucional, estas cuatro evaluaciones generales habían sido capaces de generar todo un estilo nuevo de planificación estratégica de la 
investigación por parte de las instituciones evaluadas y, todo ello, pese a las grandes controversias que van generando en el seno de la comunidad académica (Wilsdon et al., 2015). Al objeto de ir integrando novedades que permitan suavizar esas críticas, tras el RAE 1996 se elaboró una extensa consulta a los grupos participantes que dio como resultado la necesidad de favorecer la autonomía entre áreas de conocimiento y potenciar la interdisciplinariedad. Se pretendía crear un sistema más justo, consciente de la diversidad epistemológica entre los campos del saber, y, al mismo tiempo, potenciar la interdisciplinariedad como vía para alcanzar resultados con mayor impacto internacional.

En este sentido, las novedades más relevantes que incorpora el RAE 2001 son:

- Independencia de los paneles respecto a sus criterios de puntuación.

- Se establecieron grupos de discusión entre los evaluadores categorizados por grandes ramas de conocimiento (medical and biological sciences, physical sciences and engineering, social sciences, area studies and language y humanities and arts) al objeto de debatir sobre cómo practicar las evaluaciones de forma homogénea en todas las áreas sin crear desigualdades o disonancias por áreas de conocimiento. Estos grupos recibieron el nombre de «umbrella group».

- Uno de los objetivos fue ayudar a los paneles a potenciar la consistencia de la investigación, específicamente sobre la investigación interdisciplinar. Para ello, contaron con el asesoramiento de cinco expertos internacionales al objeto de valorar los resultados provisionales teniendo como referente la excelencia internacional.

Hasta 2001, ya habían transcurrido más de 25 años desde la aplicación del sistema "performativo» de evaluación y financiación. Al mismo tiempo se iba desarrollando todo un campo nuevo de investigación crítica interdisciplinar denominado «research evaluation». Este nacía en el ámbito de las ciencias sociales, al calor de los efectos y de las consecuencias de la implantación de estos macromodelos de evaluación científica. A nivel disciplinar, estos estudios se encuentran entre los límites de la sociología, la educación, la comunicación o el derecho, entre otros. En definitiva, es un fenómeno abordable desde múltiples perspectivas de estudio.

En 2008 se practicó otro RAE con la introducción de nuevos aspectos que vendrían en parte a contestar a las críticas anteriormente apuntadas. Entre las más importantes destacaron la introducción de un perfil de calidad que permitía identificar a aquellos investigadores sobresalientes en departamentos de bajo nivel, dando así respuesta a las demandas sobre la valoración de las islas de excelencia. Estos departamentos recibían un extra en el presupuesto asignado en relación a estos perfiles de calidad (quality profile). El cambio en la forma de puntuar también favoreció que las evaluaciones fueran más precisas y coherentes en términos generales con todo el personal investigador que integraba los departamentos. 
Se pasó de ocho niveles a cuatro. De esta manera, además de poder valorar a los investigadores más notables mediante el perfil de calidad, se evitaba que departamentos con una media alta ocultaran a investigadores de bajo nivel. Este nuevo método tuvo efectos un poco más equilibrados y democratizadores en términos de financiación. Es decir, la financiación, en general, fue más repartida entre todas las instituciones de educación superior (Stern, 2016).

Otra novedad bastante aplaudida fue la introducción de un nuevo indicador denominado «ecosistema académico» (research environment). EI REF explicaba que para evaluar el entorno o ecosistema académico hay que hacerlo en «términos de su vitalidad y sostenibilidad, abarcando la estrategia, a las personas, la infraestructura, los ingresos, la colaboración y la contribución de la disciplina» (REF, 2011, p. 6).

El concepto de «ecosistema académico» que acabamos de mencionar se refiere a los elementos que hacen posible un entorno óptimo para el desarrollo de la investigación: estructura social, infraestructuras materiales y políticas específicas sobre investigación.

\author{
El concepto de «ecosistema \\ académico» se refiere a los \\ elementos que hacen posible un \\ entorno óptimo para el desarrollo \\ de la investigación: estructura social, \\ infraestructuras materiales y políticas \\ específicas sobre investigación
}

\section{REF 2014: la introducción de las métricas y la medición del impacto de la ciencia}

Conforme el proceso evaluativo se fue consolidando, estos ejercicios se transformaron en una empresa muy cara para el Estado y de enorme complejidad en cuanto a la organización y logística. Para ahorrar costes, la Administración intentó implantar desde 2005 un sistema de evaluación basado en las métricas como medida alternativa al masivo peer review que supone una evaluación de estas dimensiones (Martin, 2011).

Esta propuesta ${ }^{5}$ provenía inicialmente de Her Majesty's Treasury $(\mathrm{HMT})^{6}$ y fue dirigida por el HEFCE. Consistía en la introducción de las métricas como principal medida de valoración

5 En el ordenamiento jurídico británico, white paper equivale a un documento a medio camino entre un «libro blanco» y una "proposición de ley». No se puede establecer un símil preciso con el ordenamiento jurídico español. Consiste en una proposición de debate previo a una proposición de ley. El objetivo es que la Administración proponga una solución concreta, que esta se estudie y que se produzcan debates con los miembros afectados de la sociedad. Si es aceptada, desemboca en nueva propuesta en forma de green paper. En el siguiente enlace se puede consultar la propuesta del HMT sobre la introducción de métricas como herramienta más barata respecto a la evaluación científica: <http://webarchive.nationalarchives.gov.uk/+/http:/www.hm-treasury.gov.uk/media/7/8/bud06_science_332v1.pdf>.

6 Principal órgano financiador de la ciencia en el Reino Unido. El homólogo español sería el Ministerio de Economía y Empresa. 
de la calidad científica, desarrollando un nuevo modelo general de evaluación pensado para sustituir al RAE tras el ejercicio 2008. Ante la enorme suspicacia que despertó esta propuesta, el HEFCE realizó una consulta formal a todos los miembros de la comunidad académica en 2007 y se introdujeron algunos cambios sustanciales en el planteamiento inicial, estableciendo que «habría una aproximación diferenciada por grupos de disciplinas» (Stern, 2016, p. 43).

Los resultados de la consulta realizada evidenciaban que ciertas disciplinas, como las provenientes de las ciencias naturales, son más proclives a ser evaluadas bajo el paraguas de estos indicadores métricos. Por el contrario, será mucho más complicado, impreciso o ineficaz evaluar la calidad de un output proveniente de las ciencias sociales o humanas bajo estos parámetros.

En conclusión, se admitía un uso ponderado de las métricas en función de la disciplina que se quisiera evaluar que se aplicaría en una prueba piloto en $2009^{7}$.
Los resultados de la consulta realizada evidenciaban que ciertas disciplinas, como las provenientes de las ciencias naturales, son más proclives a ser evaluadas bajo el paraguas de estos indicadores métricos. Por el contrario, será mucho más complicado, impreciso o ineficaz evaluar la calidad de un output proveniente de las ciencias sociales o humanas bajo estos parámetros

Los resultados de esta prueba consolidaron el rechazo general de la comunidad académica frente a la introducción de las métricas en detrimento de las revisiones por pares, aunque reconociendo cierto margen para su introducción como elemento complementario y dando lugar a una nueva forma de evaluación denominada «informed peer review» (Stern, 2016).

\section{El informed peer review se caracteriza por el uso de técnicas bibliométricas para complementar y asesorar las decisiones tomadas por los paneles de expertos} corporaron únicamente 11 de los 36 subpaneles (unit of assessment); fundamentalmente, aquellas disciplinas que por su desarrollo interno entendían que eran más susceptibles de ser valoradas por este sistema de indicadores bibliométricos, como, por ejemplo, medicina, química, biología o ingeniería informática.

\footnotetext{
7 El informe final se puede consultar en el siguiente enlace: <http://www.hefce.ac.uk/media/hefce/content/ pubs/2009/rd1409/rd14_09.pdf>.
} 
Otro elemento fundamental y novedoso que se introdujo en el REF 2014 fue la evaluación del impacto de la ciencia. La principal cuestión que surgió fue la falta de una metodología consolidada capaz de valorar y analizar el impacto real de la ciencia.

EI HEFCE decidió realizar una prueba piloto en $2010^{8}$ para probar el sistema de evaluación del impacto de la ciencia basado en estudios de caso. No obstante, más allá de esta prueba piloto realizada por el organismo público, la cuestión de la valoración del impacto ha abierto una gran polémica científica en torno al significado del impacto de la ciencia y su medición, sobre todo teniendo en cuenta la importancia respecto a la ponderación total, un $20 \%$ respecto al total de los méritos evaluados en 2014 y un $25 \%$ que se aplicará en el REF 2021.

El REF 2014 definía «impacto» como «un efecto, cambio o beneficio para la economía, la sociedad, la cultura, la política o los servicios públicos, la salud, el medioambiente o la calidad de vida, más allá de la academia» (REF, 2011, p. 48). Para Stern (2016), «el impacto no se centraría únicamente en la comercialización, sino que se extendería a los cambios y beneficios para la economía, la sociedad, la cultura, las políticas o servicios públicos, la salud, el medioambiente o la calidad de vida» (p. 43).

Hasta este punto del estudio hemos descrito el contexto general del sistema de evaluación científico británico, incluyendo la última prueba celebrada, denominada «REF 2014». En esta destacaron dos novedades fundamentales no exentas de polémica:

- La introducción de las métricas como elemento informador de la revisión por pares (informed peer review).

- La evaluación del impacto de la ciencia.

Ninguna de las dos novedades introducidas ha servido para facilitar el proceso o para ahorrar costes, ya que el gasto del REF 2014 superó los 247 millones de libras (Stern, 2016).

\section{Resultados y críticas}

Realizar una aproximación a los modelos tradicionales de financiación es esencial para situar cualquier investigación sobre los SEI en un marco de comprensión lógico.

8 La prueba piloto se celebró durante 2010. Para ampliar resultados, véase el siguiente enlace: <https:// www.ref.ac.uk/2014/about/background/pilot/>. 
A partir de los documentos trabajados, es posible trazar de forma sintética las diferencias entre los dos principales sistemas de financiación y evaluación de la investigación:

- Modelo «performativo».

- Modelo institucional.

Estos dos modelos se sitúan en los polos de un arco hipotético de posibilidades. La mayoría de los países optan por modelos mixtos, adaptados a sus condiciones específicas, con clara prevalencia del modelo «performativo" sobre el institucional, derivado de las recomendaciones sobre políticas científicas de entidades supranacionales (véase cuadro 1).

Cuadro 1. Diferencias entre el modelo «performativo" y el modelo institucional

Modelo «performativo»

Carácter retributivo: vinculado a resultados.

Financiación ex post.

Naturaleza competitiva: basada en la concurrencia de actores.
Modelo institucional

Carácter sustentador: vinculado a sostener las estructuras sociales.

Financiación ex ante.

Naturaleza no competitiva: basada en criterios orgánicos.

Por definición, el modelo «performativo» está basado en resultados, tiene una marcada naturaleza competitiva y un carácter descentralizador, y se realiza mediante una evaluación ex post (Hicks, 2012). La competitividad se produce cuando las diferentes unidades se presentan a la evaluación y compiten entre sí. En función de los resultados obtendrán mayor o menor financiación. Además, la competencia puede darse entre departamentos, facultades y universidades en general, ya que los sistemas «performativos» tienden a diversificar las unidades evaluables, favoreciendo la descentralización organizativa y la autonomía. En sentido contrario, el modelo institucional se realiza mediante un plan previo de financiación que está basado en criterios orgánicos, como puede ser el número de estudiantes, profesores, etc. No es competitivo, dado que la financiación se realiza en bloque, por institución en general, de ahí el carácter sustentador (Zacharewicz et al. (2019).

En el marco del EEl, la mayoría de los países, siguiendo las recomendaciones de la Comisión Europea (2017) y de la OCDE (2010), han ido introduciendo medidas «performativas» en 
sus respectivos sistemas de financiación y evaluación de la investigación, sin embargo, España se mantiene como una de las pocas excepciones al respecto, conservando un modelo institucional ${ }^{9}$ (Jonkers y Zacharewicz, 2016).

Aunque, como se ha indicado anteriormente, la mayoría de los países optan por modelos mixtos, el caso del Reino Unido sirve para ilustrar algunas de las características mencionadas del modelo «performativo» puro, al ser un país pionero en la implantación de estos sistemas. Los datos extraídos permiten sintetizar las principales características del modelo británico (véase cuadro 2).

\section{Cuadro 2. Características del modelo «performativo» británico}

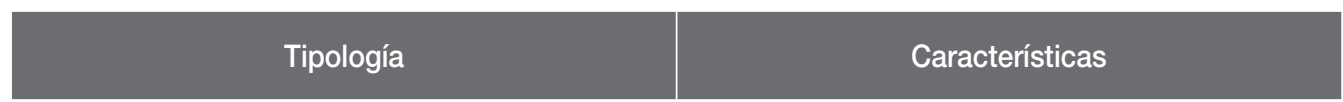

Sobre el modelo de financiación

Sistema dual con preeminencia performativa.

Sobre la metodología

Informed peer review.

Sobre la naturaleza de los actores que participan

Públicos y privados.

Abarca toda la producción científica.

Sobre el alcance y las dimensiones

Alto coste y complejidad organizativa.

Sobre el paradigma evaluativo dominante

Respecto a la producción científica, se valora más la calidad que la cantidad.

Sobre los indicadores

Múltiples (outputs, entorno y casos de impacto).

Sobre el foco de la investigación

Se potencia la interdisciplinariedad como camino hacia la excelencia internacional.

Fuente: elaboración propia.

Hasta la fecha, el Reino Unido ha celebrado siete evaluaciones generales de su investigación (1986, 1989, 1992, 1996, 2001, 2008 y 2014). La próxima evaluación se celebrará en el año 2021.

9 Según un informe realizado a instancias de la Comisión Europa por los autores referidos, las excepciones son Bulgaria, Suiza, Chipre, Grecia, Hungría, Irlanda, Luxemburgo, Letonia, Malta, Rumanía, Eslovenia y España. 
El sistema de financiación dual hace referencia a instituciones y momentos diferentes:

- Ex post. Dirigido a premiar la investigación de calidad, basado en resultados extraídos del REF y financiado por el departamento de investigación e innovación (United Kingdom Research and Innovation).

- Ex ante. Carácter prospectivo, dirigido mediante programas específicos y dependiente de los consejos de investigación (RC).

En términos globales, tiene mucho más peso la financiación que depende del REF que la basada en programas específicos de investigación (Jonkers y Zacharewicz, 2016).

Según los documentos trabajados, la introducción paulatina de las métricas se debe a un intento de rebajar el coste y la complejidad que conlleva el REF (Stern, 2016). Sin perjuicio de que algunos autores encuentren externalidades positivas en esta técnica y aboguen por su defensa, entre otros Moed (2007), Abramo y D’Angelo (2011), Hicks (2012) y Derrick y Pavone (2013), en cualquier caso, la información contrastada en las normas que regulan el REF y en las guías editadas por las instituciones hablan siempre de atemperar el uso de las métricas en función del área disciplinar que se vaya a evaluar.

Hasta el RAE 1996, la cantidad de outputs presentados fue una medida fundamental para la evaluación. Tras esa prueba, se abandonó definitivamente aquel enfoque, centrándose exclusivamente en la calidad de las aportaciones (Bence y Oppenheim, 2005).

En relación a la diversidad de los indicadores, se entiende por output cualquier producto derivado de la actividad científica en cualquier orden. Esto es, no solo producción académica escrita en forma de artículo científico, libro o cualquier soporte, sino también inventos, artefactos, patentes, obras de arte, exposiciones, informes, etc. La definición es amplia y no exhaustiva (REF, 2011; REF, 2019). Las dos principales novedades del REF 2014, y que se mantendrán para el REF 2021, son los casos de impacto y la evaluación de la ciencia.

En relación al ecosistema académico (research environment), este concepto se refiere a los elementos que hacen posible un marco óptimo para la investigación: infraestructuras materiales, estructura social y políticas específicas sobre investigación. Sobre la evaluación del impacto de la ciencia, se entiende por «impacto» cualquier beneficio para la sociedad derivado de la actividad investigadora y no focalizado exclusivamente en la actividad económica (Stern, 2016).

La investigación interdisciplinar se percibe como un elemento necesario hacia la excelencia internacional, de la misma manera que arroja retos respecto a su evaluación (REF, 2019).

Aunque la consolidación del modelo basado en resultados es un hecho en el Reino Unido tras más de 30 años de experiencia, este sistema sigue despertando ciertas críticas entre la literatura especializada. El cuadro 3 trata de sintetizar algunos resultados encontrados a partir de la investigación documental realizada. 
Cuadro 3. Aproximación a la crítica sobre los resultados derivados de la implantación del modelo «performativo»

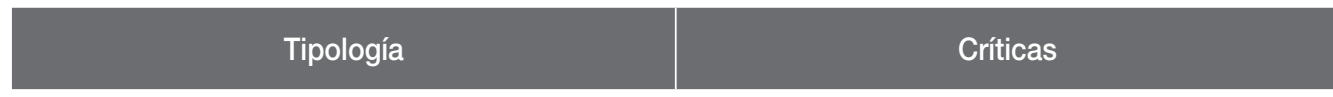

Favoreciendo el statu quo en detrimento de las ins-

Sobre la financiación tituciones emergentes (McNay, 2003; Wilsdon et al., 2015)

Inflación de la investigación: mucha cantidad y descenso de nivel (Bence y Pppenheim, 2005; Marginson, 2015; Johnson, 2017).

Sobre la praxis científica

Mala praxis (Geuna y Martin, 2003; Watermeyer y Olssen, 2016; Butler y Spoelstra, 2018).

Carreras investigadoras dirigidas a la producción en revistas de alto impacto (Barker, 2007).

Sobre la organización

Coste muy alto y gran movilización de recursos humanos (REF, 2011; REF, 2019).

Confusión entre «impacto de la ciencia» y «factor de impacto» (Watermeyer y Olssen, 2016).

Problemas ontológicos (Martin, 2011).

Sobre la evaluación del impacto de la ciencia

Confusión conceptual (Penfield, Baker, Scoble y Wykes, 2014).

Fácilmente manipulable (Marginson, 2015).

Fuente: elaboración propia.

Sobre el aspecto económico, algunos autores han sostenido que este modelo favorece la concentración y el reforzamiento del statu quo y no sirve para estimular el crecimiento de instituciones emergentes, dado el efecto capitalizador de las instituciones más importantes, que concentran los mejores investigadores y, por ende, obtienen los mejores resultados, generando así un ciclo que impide el desarrollo de instituciones emergentes (McNay, 2003 y 2010; Wilsdon et al., 2015).

Desde una perspectiva sociológica de análisis, otros autores destacan que tanto las instituciones como los investigadores han aprendido el funcionamiento de los ejercicios 
evaluativos y han desarrollado estrategias no transparentes, denominadas "game playing» (Watermeyer y Olssen, 2016; Butler y Spoelstra, 2018). Para explicar este fenómeno, Geuna y Martin (2003) se hacen eco de la «hipótesis de la Reina Roja», propuesta por Leigh van Valen en 1973 para el campo de las teorías evolutivas de las especies. Esta hipótesis describe cómo las especies deben adaptarse continuamente solo para permanecer en la misma posición respecto a otros sistemas con los que están coevolucionando. El efecto producido es que, como consecuencia de que los competidores ya han aprendido la forma en la que funciona el juego, es necesario correr más rápido para permanecer en la misma posición. En relación al contexto descrito, explicaría que el sistema no solo favorece, sino que también potencia el statu quo, poniendo serias trabas a cualquier disrupción que pueda generar procesos transformadores y de innovación. Esto explicaría cómo los protagonistas (académicos e instituciones) han desarrollado diferentes estrategias fraudulentas en el ámbito de la comunicación científica para alcanzar buenos resultados.

Según Barker (2007), el sistema favorece una visión unidireccional de hacer investigación: poniendo mayor énfasis en los artículos de revistas -bien posicionados en los diferentes índices de citación internacional-, obviando el valor de la docencia. Estos aspectos han tenido un impacto muy negativo en algunas disciplinas como el derecho, en la que las prácticas relacionadas con el posicionamiento de revistas científicas en los índices de citación, históricamente, no han tenido demasiado peso y el prestigio se mide por factores endógenos.

Sobre la medición del impacto de la ciencia, para Martin (2011) existen algunos problemas fundamentales en la raíz de considerar la medición del impacto de la ciencia como algo viable, tanto por el sentido del concepto en sí como por la metodología que habría que utilizar. Watermeyer y Olssen (2016) apuntan a la confusión que se genera entre impacto de la ciencia y factor de impacto, aunque los documentos oficiales del REF hagan expresa distinción entre ellos (REF, 2011; REF, 2019). Para Penfield et al. (2014), el problema es conceptual, derivado de la falta de una definición clara y precisa, y distingue entre «impacto académico», producido en el interior de un campo científico, e «impacto socioeconómico», externo al campo científico. Marginson (2015) apunta que el sentido de impacto es fácilmente manipulable.

Sobre la introducción del sistema informado de revisión por pares, algunos autores realizan una doble argumentación positiva. Por una parte vendrían a contrarrestar el sesgo individual que tiene el peer review y, de otra, la falta de contextualización de los indicadores bibliométricos (Donovan, 2007; Moed, 2007). Otros ponen el énfasis en la capacidad que presenta esta técnica para aumentar la participación de actores ajenos al mundo académico en la evaluación externa del impacto de la ciencia. Estos argumentan que puede 
ser una forma sencilla de visualizar los resultados derivados de una financiación externa o de una determinada política pública y que, en definitiva, podría facilitar una óptima sinergia entre el sector público y el privado (Derrick y Pavone, 2013).

Por último, se ha observado un aumento del porcentaje de excelencia que algunos autores han señalado como consecuencia de la tendencia progresiva a bajar el nivel de las evaluaciones al producirse una inflación general en todo el sistema universitario (Bence y Oppenheim, 2005; Jonhson, 2017).

\section{Conclusiones}

La OCDE (2010) ha remarcado la necesidad de vincular financiación a resultados y de implantar, por lo tanto, sistemas de evaluación basados en resultados. La experiencia del Reino Unido, país pionero en la implantación de sistemas «performativos» de evaluación, puede ser un espejo para el resto de países miembros de la OCDE y del EEl que no hayan avanzado todavía en esa dirección, como es el caso de España, uno de los pocos países que aún no ha progresado nada en ese modelo de financia-
La experiencia del Reino Unido, país pionero en la implantación de sistemas «performativos» de evaluación, puede ser un espejo para el resto de países miembros de la OCDE y del EEI que no hayan avanzado todavía en esa dirección, como es el caso de España ción de la investigación (Zacharewicz, 2019). Sin embargo, este modelo también ha hecho aflorar algunas críticas basadas en la incapacidad de potenciar instituciones emergentes, dado el poder capitalizador de las instituciones con más influencia (McNay, 2003 y 2010).

Sobre la metodología empleada en la evaluación, la base es la revisión por pares y nunca los diferentes productos bibliométricos. Las métricas se utilizan como herramienta complementaria y teniendo en cuenta el área disciplinar sobre el que se van a aplicar, dando lugar a

Sobre la metodología empleada en la evaluación, la base es la revisión por pares y nunca los diferentes productos bibliométricos. Las métricas se utilizan como herramienta complementaria y teniendo en cuenta el área disciplinar sobre el que se van a aplicar, dando lugar a la denominada «revisión por pares informados» la denominada «revisión por pares informados».

Esta denominación no es precisa, ni justa, en el sentido de que el punto de partida de la misma es considerar la revisión por pares como no informada, en contraposición a la informada, por incluir una perspectiva bibliométrica. Cabe argüir en contra de esta definición que la información previa de los expertos puede considerarse también como elemento complementario, a modo de bagaje y experiencia, de cara a los juicios 
de valor que realicen, sin perjuicio de las posibles deficiencias que intrínsecamente puedan observarse en la revisión por pares y que la literatura especializada continúa investigando.

Sobre los indicadores que se usan en la evaluación del ecosistema académico, es necesario subrayar que han resultado ser esenciales para valorar el entorno donde ha de producirse una investigación de calidad que aspire a la excelencia internacional. Nos referimos a medios materiales, a infraestructuras y al factor humano. Todo ello puede crear un ambiente propicio para la producción científica o, por el contrario, una atmósfera no recomendable.

Según los datos analizados, la medición de la reputación entre pares (peer esteem) fue una medida muy valiosa que convendría poner de nuevo en valor. Los premios, la divulgación en canales no académicos, la pertenencia a edi-

La medición de la reputación entre pares (peer esteem) fue una medida muy valiosa que convendría poner de nuevo en valor toriales o a colegios profesionales, así como cualquier acto por analogía equivalente, expresan un carácter esencial de la vida de los investigadores y podrían ser considerados indicadores válidos de la calidad de los resultados.

Sobre los indicadores que se usan en la evaluación del ecosistema académico, es necesario subrayar que han resultado ser esenciales para valorar el entorno donde ha de producirse una investigación de calidad que aspire a la excelencia internacional

La interdisciplinariedad de la producción científica se ve como un camino que hay que potenciar para alcanzar la excelencia internacional

Sobre las prácticas sociales, los actores del sistema (investigadores e instituciones) han aprendido a crear estrategias no transparentes para obtener buenos resultados. Estas prácticas son conocidas como «game playing». Los efectos de esta mala praxis en la comunicación científica constituyen un campo muy amplio de estudio que debe ser abordado desde diferentes perspectivas interdisciplinares. 
A pesar de las críticas analizadas, el sistema «perfomativo» de evaluación de la investigación, o sistema de evaluación y financiación basado en resultados, ha ofrecido externalidades muy positivas al sistema de investigación británico, situándolo como uno de los países más dinámicos en el campo de la producción científica. Los estudios de marcos comparados pueden funcionar como base para el desarrollo de estudios de naturaleza empírica más profunda y con un enfoque más práctico. Ahondar en esta materia puede facilitar la transición entre modelos, generando una información muy válida para los diferentes operadores jurídicos. En el caso de España, la implantación de un modelo de estas características que sirva para homogeneizarse con el EEl ha de pasar por una transformación del modelo clásico de financiación por bloques a un modelo que ofrezca mayor autonomía y flexibilidad a las diferentes entidades que concurren en el sistema de investigación.

\section{Referencias bibliográficas}

Abramo, G. y D'Angelo, C. A. (2011). Evaluating research: from informed peer review to bibliometrics. Scientometrics, 87(3), 499-514.

Barker, K. (2007). The UK research assessment exercise: the evolution of a national research evaluation system. Research Evaluation, 16(1), 3-12.

Bauer, M. y Kogan, M. (1997). Evaluation systems in the UK and Sweden: successes and difficulties. European Journal of Education, 32(2), 129-143. Recuperado de <https://www.jstor.org/stable/1503544 ?seq=1\#page_scan_tab_contents> (consultado el 21 de junio de 2019).

Bence, V. y Oppenheim, C. (2005). The evolution of the UK's research assessment exercise: publications, performance and perceptions. Journal of Educational Administration and History, 37(2), 137-155.

Butler, N. y Spoelstra, S. (2018). Academics at play: why the "Publication Game» is more than a metaphor. Academy of Management Proceedings, 1.
Comisión Europea. (2017). ERA Progress Report 2016. Directorate General for Research and Innovation.

Denzin, N. K. (1970). The Research Act: A Theoretical Introduction to Sociological Methods. London: Butterworths.

Denzin, N. K. y Lincoln, S. (2011). The Sage Handbook of Qualitative Research. (4. ${ }^{\mathrm{a}}$ ed.). London: Sage.

Derrick, G. E. y Pavone, V. (October 2013). Democratising research evaluation: achieving greater public engagement with bibliometrics-informed peer review. Science and Public Policy, 40(5), 563-575.

Donovan, C. (October 2007). The qualitative future of research evaluation. Science and Public Policy, 34(8), 585-597.

García Fernández, D. (2015). La metodología de la investigación jurídica en el siglo XXI. En W. A. Godínez Méndez y J. H. García Peña (Coords.), Metodologías: enseñanza e investigación jurídica (pp. 449-466). México: Universidad Nacional Autónoma de México. 
Geuna, A. y Martin, B. R. (2003). University research evaluation and funding: an international comparison. Minerva, 41(4), 277-304.

Gilroy, P. y McNamara, O. (2009). A critical history of research assessment in the United Kingdom and its post-1992 impact on education. Journal of Education for Teaching, 35(4), 321-335.

Hicks, D. (2012). Performance-based university research funding systems. Research Policy, $41(2), 251-261$.

Hughes, A., M., Kitson, A., Bullock, A. y Milner, I. (February 2013). The Dual Funding Structure for Research in the UK: Research Council and Funding Council Allocation Methods and the Pathways to Impact of UK Academics. A report from CBR/UK-IRC/BIS.

Johnson, J. (7 September 2017). Embracing accountability and promoting value for money in higher education. University UK Anual Conference. London.

Jonkers, K. y Zacharewicz, T. (2016). Research performance Based Funding Systems: a Comparative Assessment. Publications Office of the European Union, Luxembourg.

Marginson, S. (2015). UK research excellence: getting better all the time? International Higher Education, 81, 10-12.

Martin, B. R. (September 2011). The research excellence framework and the «impact agenda»: arewe creating aFrankenstein monster?, Research Evaluation, 20(3), 247-254.

McNay, I. (February 2003). Assessing the assess ment: an analysis of the UK research assessment exercise and its outcomes, with special reference to research in education. Science and Public Policy, 30(1), 47-54.

McNay, I. (2010). Research quality assessment. En P. Peterson, E. Baker y B. McGaw (Eds.), International Encyclopedia of Education (pp. 307-315). Oxford, UK: Elsevier Ltd.
Moed, H. F. (October 2007). The future of research evaluation rests with an intelligent combination of advanced metrics and transparent peer review. Science and Public Policy, 34(8), 575-583.

OCDE (2010). Performance-Based Funding for Public Research in Tertiary Education Institutions: Workshop Proceedings. OECD Publishing.

Osuna López, C. (2010). Evaluando el impacto del sistema de evaluación español sobre la producción científica nacional (1980-2005): laaplicación deun diseñocuasiexperimental, En H. Vessuri, P. Kreimer, A. Arellano y L. Sanz, Conocer para transformar (pp. 223248). Caracas: IESALC-UNESCO.

Penfield, T., Baker, M. J., Scoble, R. y Wykes, M. C. (January 2014). Assessment, evaluations, and definitions of research impact: a review. Research Evaluation, 23(1), 21-32.

RAE. (1992). The 1992 Research Assessment Exercise. Recuperado de <http://www.rae. ac.uk/1992/index.html> (consultado el 21 de junio de 2019).

RAE. (1996a). The 1996 Research Assessment Exercise. Recuperado de <http://www.rae. ac.uk/1996/index.html> (consultado el 21 de junio de 2019).

RAE. (1996b). The 1996 Research Assessment Exercise. RAE96 1/94. Recuperado de <https: //www.rae.ac.uk/1996/c1_94.html> (consultado el 21 de junio de 2019).

RAE. (2001). The 2001 Research Assessment Exercise. Recuperado de <http://www.rae. ac.uk/2001/> (consultado el 21 de junio de 2019).

RAE. (2008). The 2008 Research Assessment Exercise. Recuperado de <http://www.rae. ac.uk/> (consultado el 21 de junio de 2019).

REF. (2011). REF 2014. Assessment framework and guidance on submissions. REF 02.2011. Recuperado de <https://www.ref. ac.uk/2014/pubs/2011-02/> (consultado el 21 de junio de 2019). 
REF. (2019). REF 2021. Guidance on Submissions. REF2019/01. Recuperado de $<$ https://www.ref.ac.uk/media/1092/ref2019_01-guidance-on-submissions.pdf> (consultado el 21 de junio de 2019).

Stern, L. N. (2016). Research Excellence Framework (REF) Review: Building on Success and Learning from Experience. Ref. IND/ 16/9. Department for Business, Energy and Industrial Strategy.

Watermeyer, R. y Olssen, M. (2016). «Excellence» and exclusion: the individual costs of institutional competitiveness. Minerva, 54(2), 201-218. Recuperado de <https:// link.springer.com/article/10.1007\%2Fs11 024-016-9298-5> (consultado el 25 de mayo de 2019).
Wilsdon, J., Allen, L., Belfiore, E., Campbell, P., Curry, S., Hill, S., ... y Johnson, B. (2015). The Metric Tide: Report of the Independent Review of the Role of Metrics in Research Assessment and Management.

Witker, J. (2008). Hacia una investigación jurídica integrativa. Boletín Mexicano de Derecho Comparado, 41(122), 943-963. Recuperado de https://revistas.juridicas. unam.mx/index.php/derecho-comparado/ article/view/3996 (consultado el 21 de junio de 2019).

Zacharewicz, T., Lepor, B., Reale, E. y Jonkers, K. (February 2019). Performance-based research funding in EU Member States-a comparative assessment. Science and Public Policy, 46(1), 105-115.

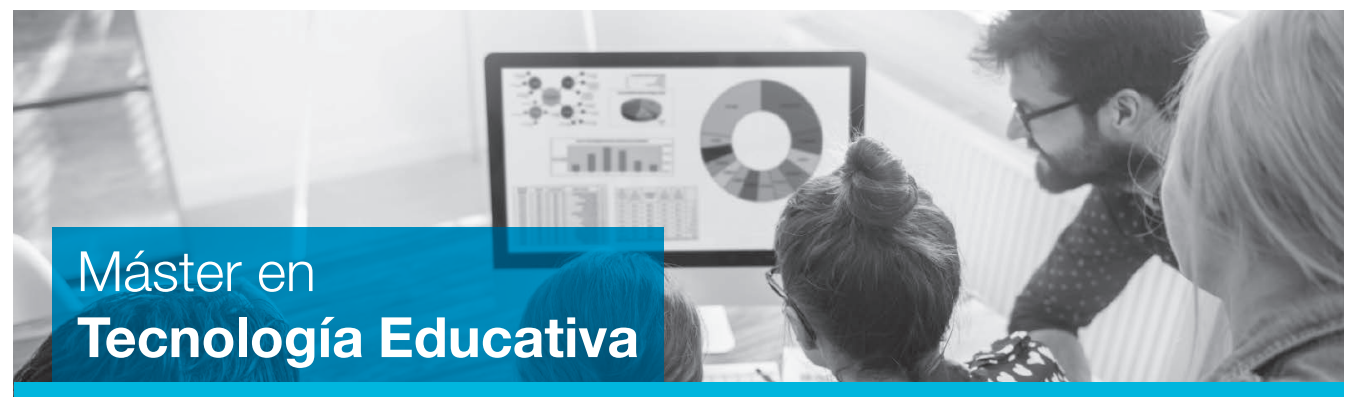

Este máster oficial [60 créditos ECTS] tiene una duración normal de 12 meses.

Dirigido a: Titulados universitarios de las distintas ramas del conocimiento que deseen especializarse en el correcto desempeño de las funciones de un experto en tecnología educativa. No exige experiencia previa en el ámbito educativo. Especialmente dirigido a titulados en Magisterio, Pedagogía y Educación Social.

Aquellas personas interesadas que no provengan de las titulaciones anteriormente citadas deberán realizar unos complementos formativos.

Objetivos: Capacitar a profesores, investigadores y educadores en el conocimiento y empleo de las nuevas tecnologías de la comunicación y la información, así como de los modelos formativos e-learning y b-learning, en beneficio de las acciones formativas en los nuevos contextos educativos. También profundiza en el conocimiento de las posibilidades que ofrecen las tecnologías actuales y emergentes para encontrar nuevas formas de obtención y manejo de información en ámbitos educativos. 
Para los Grados en Derecho o en

Ciencias del Trabajo, Relaciones

Laborales y Recursos Humanos

- Administración y Finanzas

- Asistencia a la Dirección

- Secretariado

\section{Para los Grados en Administración y}

Dirección de Empresas o en Economía

- Administración y Finanzas

- Asistencia a la Dirección

- Comercio Internacional

- Gestión Comercial y Marketing

- Marketing y Publicidad

- Secretariado

- Transporte y Logística

\section{Para el Grado en Marketing}

- Administración y Finanzas

- Comercio Internacional

- Gestión Comercial y Marketing
- Gestión de Alojamientos Turísticos

- Gestión de Ventas y Espacios Comerciales

- Marketing y Publicidad

- Transporte y Logística

\section{Para los Grados en Magisterio de Educación Infantil y en Magisterio de \\ Educación Primaria}

- Animación de Actividades Físicas y Deportivas

- Educación Infantil

- Integración Social

- Animación Sociocultural y Turística

\section{Para el Grado en Empresas} y Actividades Turísticas

- Gestión Comercial y Marketing

- Gestión de Ventas y Espacios Comerciales

- Gestión de Alojamientos Turísticos

- Agencias de Viajes y Gestión de Eventos

- Guía, Información y Asistencias Turísticas

\footnotetext{
* De acuerdo a lo establecido en el Real Decreto 1618/2011, de 14 de noviembre, sobre reconocimiento de estudios en el ámbito de la educación superior.
} 


\section{¿Qué nos diferencia de otras Universidades online?}

La Universidad a Distancia de Madrid, UDIMA, está diseñada para cubrir las necesidades de las personas del siglo XXI: profesionales que demandan una titulación universitaria reconocida oficialmente y de prestigio, adaptada a Europa y en contacto con el mundo de la empresa, y que facilite, además, una buena inserción laboral o mejore la que ya se posee.
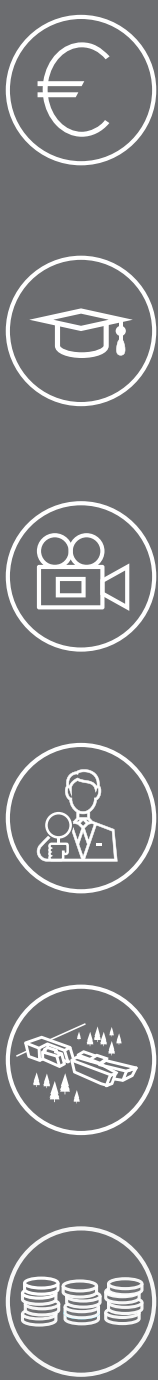

\section{Pago fraccionado}

Para que el dinero no sea un impedimento, te ofrecemos la posibilidad de realizar el pago fraccionado o a través de financiación bancaria. Que estudiar sea tu única preocupación.

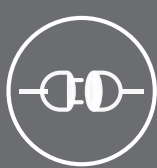

\section{Siempre conectados}

Nuestro compromiso es contestar en menos de $48 \mathrm{~h}$ a todas tus dudas, para que cumplir tus objetivos te sea más fácil. Siempre estaremos conectados.

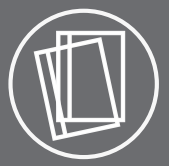

\section{Materiales adaptados}

Contamos con una Editorial propia que desarrolla los libros y carpetas especialmente diseñados para el aprendizaje online, que te llegarán a casa al principio de cada semestre.

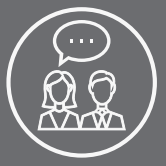

\section{Encuentros presenciales}

Realizamos talleres, conferencias y prácticas presenciales voluntarias que amplían el contenido de las asignaturas.

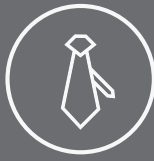

\section{Contacto con empresas}

Nuestra Bolsa de Trabajo y

Emprendedores te ofrece asesoría individualizada para que puedas potenciar tus cualidades y posicionarte como quieras en el mercado laboral.

\section{Sedes de examen}

Estamos cerca de ti. Además de alrededor de toda España, contamos con sedes en Europa, Asia y

América, con especial relevancia en Latinoamérica.

\section{Convocatoria en septiembre}

No es fácil compaginar el estudio con la vida personal y profesional. Por eso tenemos una convocatoria extra en septiembre. Tienes dos oportunidades al año de aprobar cada asignatura. 